\title{
CUT-OFF AND HITTING TIMES OF A SAMPLE OF ORNSTEIN-UHLENBECK PROCESSES AND ITS AVERAGE
}

\author{
B. LACHAUD, ${ }^{*}$ Université René Descartes - Paris 5
}

\begin{abstract}
A cut-off phenomenon is shown to occur in a sample of $n$ independent, identically distributed Ornstein-Uhlenbeck processes and its average. Their distributions stay far from equilibrium before a certain $O(\log (n))$ time, and converge exponentially fast after. Precise estimates show that the total variation distance drops from almost 1 to almost 0 over an interval of time of length $O(1)$ around $\log (n) /(2 \alpha)$, where $\alpha$ is the viscosity coefficient of the sampled process. The distribution of the hitting time of 0 by the average of the sample is computed. As $n$ tends to infinity, the hitting time becomes concentrated around the cut-off instant, and its tails match the estimates given for the total variation distance.
\end{abstract}

Keywords: Cut-off; hitting time; Ornstein-Uhlenbeck process

2000 Mathematics Subject Classification: Primary 60J60

\section{Introduction}

The cut-off phenomenon has been widely investigated in the past twenty years; see, for instance, [5] and [14] for general references. It describes the property of steep convergence to equilibrium of certain Markov processes. Before the so-called 'cut-off instant', they remain as far apart as possible, at least in the sense of the total variation distance. After the cut-off instant, they converge exponentially fast (see Definition 2). This phenomenon has been recognized in various families of Markov processes or chains; see [6], [15], and [18], for example. In [17], Ycart showed that a cut-off occurs for samples of Markov chains on a finite set, both in discrete and continuous time. This result has recently been extended in [2] to exponentially converging processes.

The aim of this paper is to investigate the cut-off phenomenon for a sample of OrnsteinUhlenbeck (OU) processes and its average process. The Ornstein-Uhlenbeck process has been widely studied in the past seventy years. It was first introduced in physics in 1930 [16], but has also been used, for example, in financial mathematics to model prices in markets [9] and in biology to model neural activity [10].

The interest of OU processes is that almost all computations can be carried out explicitly. In particular, precise estimates of the distance between the distribution of the sample at time $t$ and its asymptotic distribution as $t$ tends to infinity can be given (see Proposition 1). The empirical mean of $n$ independent, identically distributed (i.i.d.) OU processes is another OU process. Thus, it is possible to compute the distance to equilibrium also for the average process. Its turns out that the asymptotics is the same as for the sample (see Proposition 2).

Received 21 March 2005; revision received 11 May 2005.

* Postal address: MAP5, UMR CNRS 8145, Université Paris 5, 45 rue des Saints-Pères, 75270 Paris Cedex 06, France.

Email address: beatrice.lachaud@math-info.univ-paris5.fr 
The study of hitting times of Ornstein-Uhlenbeck processes is also possible. Various methods for computing the distribution of hitting times of diffusion processes are available [3, Chapter 3.4], [8, p. 475]. In the case of the OU process, a series expression for the density of the hitting time of a fixed level has been proposed by Pitman and Yor [12]. When that level is 0 , an explicit expression of the density is obtained. We shall apply this result to the average process of a sample of $n$ OU processes and deduce the asymptotics of the hitting time as $n$ tends to infinity (see Proposition 3). This asymptotic distribution can be viewed as that of the logarithm of the absolute value of a Gaussian random variable, and its tail behavior can be studied precisely. Interestingly, it turns out that the asymptotics of the distance to equilibrium for both the sample and the average coincide with the tail behaviors of the hitting time.

The article is organized as follows. In Section 2, we recall the necessary background on the one-dimensional OU process and on the cut-off phenomenon. The cut-off phenomenon and the precise estimates for the distances between the distribution at time $t$ and the asymptotic distribution as $t$ tends to infinity are studied in Section 3, first for the sample and then for the average process. The hitting time of the average process and the link with the cut-off phenomenon are the subject of Section 4.

\section{Framework and preliminaries}

In this section, we review the necessary background, first on the OU process and then on the cut-off phenomenon.

\subsection{Some useful properties of the one-dimensional OU process}

The OU process appears as the solution of the so-called Langevin equation, a stochastic differential equation that models the movement of a Brownian particle in a viscous fluid. It is a diffusion process with linear drift and constant diffusion coefficient. The precise definition is as follows.

Definition 1. Let $\alpha>0, \sigma>0$, and $x_{0}$ be real numbers. Let $(B(t))_{t \geq 0}$ be a standard Brownian motion. We call the unique solution of the stochastic differential equation

$$
\mathrm{d} X(t)=-\alpha X(t) \mathrm{d} t+\sigma \sqrt{2 \alpha} \mathrm{d} B(t), \quad X(0)=x_{0},
$$

an OU process starting at $x_{0}$ with parameters $\alpha$ and $\sigma$.

Now consider an OU process $X$ starting at $x_{0}$ with parameters $\alpha$ and $\sigma$. The process $X$ can be explicitly written as

$$
X(t)=x_{0} \mathrm{e}^{-\alpha t}+\sigma \sqrt{2 \alpha} \int_{0}^{t} \mathrm{e}^{-\alpha(t-u)} \mathrm{d} B(u) .
$$

Consequently this process is Gaussian, with mean

$$
\mathrm{E}(X(t))=x_{0} \mathrm{e}^{-\alpha t}
$$

and covariance function

$$
\operatorname{cov}(X(s), X(t))=\sigma^{2}\left[\mathrm{e}^{-\alpha|t-s|}-\mathrm{e}^{-\alpha(t+s)}\right] .
$$

In particular, the distribution of $X(t)$ is Gaussian with mean $x_{0} \mathrm{e}^{-\alpha t}$ and variance $\sigma^{2}\left(1-\mathrm{e}^{-2 \alpha t}\right)$. Hence, the stationary distribution $v$ of the process $X$ is the centered Gaussian distribution with 
variance $\sigma^{2}$, denoted by $\mathcal{N}\left(0, \sigma^{2}\right)$. Finally, the OU process $X$ converges to its stationary distribution as $t \rightarrow \infty$, i.e.

$$
X(t) \stackrel{\mathrm{D}}{\rightarrow} \mathcal{N}\left(0, \sigma^{2}\right) .
$$

More precisely, this convergence takes place at an exponential rate. The total variation distance between the distribution of $X$ at time $t$ (denoted by $\mathcal{L} X(t)$ ) and its stationary distribution $v$ has the following form as $t$ tends to infinity (see Appendix A for a proof):

$$
\|\mathcal{L} X(t)-v\|_{\mathrm{TV}}=\frac{\left|x_{0}\right|}{\sigma \sqrt{2 \pi}} \mathrm{e}^{-\alpha t}+o\left(\mathrm{e}^{-2 \alpha t}\right) .
$$

Let us now consider the hitting time $T_{0}$ of the level 0 by the OU process $X$,

$$
T_{0}=\inf \{t \geq 0: X(t)=0\} .
$$

We are interested in the level 0 because zero is the expected value of the stationary distribution of the process $X$, and we will see in Section 4 that the cut-off phenomenon can be linked to the hitting time of the expected value of the stationary distribution using an empirical process.

A study of the hitting time $T_{0}$, in the more general case in which 0 is replaced by any fixed level $a$, has already been made [12]. It is used in several applications; for example, in [11] the distribution of the hitting time was needed to find a pricing formula for interest rate pathdependent options in financial models. A survey on the use of the hitting time of an OU process can be found in [1]. In the general case, its density $f$ is given by a series expansion, while, when the level is 0 , the density can be computed explicitly [12], [1]:

$$
f(t)=\frac{\left|x_{0}\right|}{2 \sigma \sqrt{\pi \alpha}}\left(\frac{\alpha}{\sinh (\alpha t)}\right)^{3 / 2} \exp \left(-\frac{x_{0}^{2} \mathrm{e}^{-\alpha t}}{4 \sigma^{2} \sinh (\alpha t)}+\frac{\alpha t}{2}\right) .
$$

\subsection{The cut-off phenomenon}

The cut-off phenomenon is described here in terms of the total variation distance. Recall that this distance is defined between two probability distributions $\mu$ and $v$ on a common space $E$ by

$$
\|\mu-v\|_{\mathrm{TV}}=\sup _{A \subset E}|\mu(A)-v(A)| .
$$

The cut-off phenomenon is defined for a sequence of stochastic processes as follows.

Definition 2. Suppose that, for each $n \in \mathbb{N}$, we have

- a measurable space $E^{(n)}$ and

- a stochastic process $X^{(n)}=\left\{X^{(n)}(t), t \geq 0\right\}$, with values in $E^{(n)}$, that converges to a distribution $v^{(n)}$ on $E^{(n)}$ as $t$ tends to $\infty$.

For $t \geq 0$, we define $d^{(n)}(t)=\left\|\mathcal{L} X^{(n)}(t)-v^{(n)}\right\|_{\mathrm{TV}}$, where $\mathcal{L} X^{(n)}(t)$ is the distribution of $X^{(n)}$ at time $t$. Let $\left(t_{n}\right)_{n \in \mathbb{N}^{*}}$ be a nondecreasing deterministic sequence of times. It is called a sequence of cut-off times if, for $c>0$,

$$
\begin{aligned}
& c<1 \Rightarrow \lim _{n \rightarrow \infty} d^{(n)}\left(c t_{n}\right)=1, \\
& c>1 \Rightarrow \lim _{n \rightarrow \infty} d^{(n)}\left(c t_{n}\right)=0 .
\end{aligned}
$$


Note that, for a Markov process $X$ that converges to its stationary distribution $v$, the total variation distance between the distribution of $X$ at time $t$ and the distribution $v$ is a nonincreasing function of $t$. This implies, in particular, that a sequence of cut-off times is not unique. If $\left(t_{n}\right)_{n \in \mathbb{N}^{*}}$ is a sequence of cut-off times then all sequences $\left(\tau_{n}\right)_{n \in \mathbb{N}^{*}}$ equivalent to $\left(t_{n}\right)_{n \in \mathbb{N}^{*}}$ (in the sense that the ratio $t_{n} / \tau_{n}$ tends to 1 ) are also sequences of cut-off times.

A particular case in which a cut-off phenomenon has already been proved to occur was studied by Ycart [17]. In that case, the process $X^{(n)}$ is a sample of $n$ i.i.d. finite-valued Markov chains that converge to their stationary distribution. The cut-off instants have the form $\log (n) / 2 \beta$, where $\beta$ is the spectral gap of the infinitesimal generator of the sampled chain. (For reversible Markov chains, the spectral gap is defined as the smallest nonzero eigenvalue of the infinitesimal generator (see [14, p. 327]).) In what follows, we will use the same ideas in the case of the OU process.

In order to bound total variation distances, we will use the Hellinger distance $H$. For two probability measures $\mu$ and $v$, with respective densities $p$ and $q$ with respect to the same measure $\lambda$, the Hellinger distance separating them is defined as

$$
H(\mu, v)=\left(\frac{1}{2} \int(\sqrt{p}-\sqrt{q})^{2} \mathrm{~d} \lambda\right)^{1 / 2}
$$

The following classical inequality holds (see [13, p. 61]):

$$
H^{2}(\mu, v) \leq\|\mu-v\|_{\mathrm{TV}} \leq H(\mu, v) \sqrt{2-H^{2}(\mu, v)} .
$$

In the case of the OU processes all distributions are Gaussian. For all integers $n$, the Hellinger distance between two samples of $n$ i.i.d. Gaussian random variables, with respective means $m_{1}$ and $m_{2}$ and respective variances $\sigma_{1}^{2}$ and $\sigma_{2}^{2}$, is given explicitly by

$$
H^{2}\left(\mathcal{N}\left(m_{1}, \sigma_{1}^{2}\right)^{\otimes n}, \mathcal{N}\left(m_{2}, \sigma_{2}^{2}\right)^{\otimes n}\right)=1-\left(\frac{2 v_{1} v_{2}}{v_{1}^{2}+v_{2}^{2}}\right)^{n / 2} \exp \left(-\frac{n\left(m_{1}-m_{2}\right)^{2}}{4\left(v_{1}^{2}+v_{2}^{2}\right)}\right) .
$$

In the next section, we study the cut-off phenomenon for a sample of $n$ i.i.d. OU processes and for its empirical mean.

\section{Cut-off for OU processes}

\subsection{Cut-off for a sample of $\boldsymbol{n}$ OU processes}

Consider a sample of $n$ i.i.d. OU processes starting at $x_{0}$ with parameters $\alpha$ and $\sigma$. At each instant $t$, the sample has an $n$-dimensional Gaussian distribution that converges, as $t$ tends to $\infty$, to another $n$-dimensional Gaussian distribution. For a positive integer $n$, let us denote by $d_{n}(t)$ the total variation distance between the distribution of the sample of OU processes $\left(X_{1}, \ldots, X_{n}\right)$ at time $t$ and its stationary distribution $v^{\otimes n}$ :

$$
d_{n}(t)=\left\|\mathcal{L}\left(X_{1}(t), \ldots, X_{n}(t)\right)-v^{\otimes n}\right\|_{\mathrm{TV}}
$$

Proposition 1. The sample $X^{(n)}=\left(X_{1}(t), \ldots, X_{n}(t)\right)$ has a cut-off at the instant

$$
t_{n}=\frac{\log (n)}{2 \alpha}
$$


and, for all sufficiently large integers $n$ and for all real $u$ such that $t_{n}+u>0$, the following inequalities hold:

$$
\begin{gathered}
1-\exp \left(-\frac{x_{0}^{2}}{8 \sigma^{2}} \mathrm{e}^{-2 \alpha u}\right)+\varepsilon_{n}(u) \leq d_{n}\left(t_{n}+u\right), \\
d_{n}\left(t_{n}+u\right) \leq \sqrt{1-\exp \left(-\frac{x_{0}^{2}}{4 \sigma^{2}} \mathrm{e}^{-2 \alpha u}\right)}+\varepsilon_{n}^{\prime}(u) .
\end{gathered}
$$

Here $\varepsilon_{n}(u)$ and $\varepsilon_{n}^{\prime}(u)$ converge to 0 as $n$ tends to infinity.

This proposition shows that the cut-off actually occurs over an interval of time of length $O(1)$, which is more precision than required in Definition 2.

This cut-off phenomenon is a particular case of the cut-off studied in [2] for general exponentially converging processes. Formula (1) says that the OU process is an exponentially converging process, in the sense that the total variation distance between its distribution at time $t$ and the stationary distribution is exponentially decreasing as $t$ tends to infinity. It was proved in [2] that all samples of $n$ exponentially converging processes exhibit a cut-off at an instant of order $\log (n)$.

Proof of Proposition 1. Let us begin with the proofs of (5) and (6). The sample $X^{(n)}$ at time $t$ has density $\mathcal{N}\left(x_{0} \mathrm{e}^{-\alpha t}, \sigma^{2}\left(1-\mathrm{e}^{-2 \alpha t}\right)\right)^{\otimes n}$ and its stationary distribution, as $t$ tends to infinity, is $\mathcal{N}\left(0, \sigma^{2}\right)^{\otimes n}$. Thus, the Hellinger distance between the distribution of $X^{(n)}$ at time $t$ and its stationary distribution can be computed using (4). Inequality (3) then bounds the total variation distance by the Hellinger distance and, finally, the study of the behavior of the upper and lower bounds as $n$ tends to infinity gives the expected results.

Let us then notice that

$$
\begin{aligned}
& \lim _{u \rightarrow-\infty} 1-\exp \left(-\frac{x_{0}^{2}}{8 \sigma^{2}} \mathrm{e}^{-2 \alpha u}\right)=1, \\
& \lim _{u \rightarrow \infty} \sqrt{1-\exp \left(-\frac{x_{0}^{2}}{4 \sigma^{2}} \mathrm{e}^{-2 \alpha u}\right)}=0,
\end{aligned}
$$

and that, for all $n$, the function $d_{n}$ is nonincreasing (as recalled in Section 2.2). Hence, we conclude that $t_{n}$ is a cut-off instant in the sense of Definition 2.

In the next section, we will see that a cut-off phenomenon is exhibited by the average process of the sample, and has the same cut-off instant.

\subsection{Cut-off for the average of $\boldsymbol{n}$ i.i.d. OU processes}

Let us now study what happens for the average process of the sample, defined at each instant as the arithmetic mean of the sample. Let $\left(X_{1}, \ldots, X_{n}\right)$ be a sample of $n$ OU processes starting at $x_{0}$ with parameters $\alpha$ and $\sigma$. We denote by $M_{n}$ the average process,

$$
M_{n}(t)=\frac{1}{n} \sum_{i=1}^{n} X_{i}(t), \quad t \geq 0 .
$$

The process $M_{n}$ is a particular linear combination of $n$ independent OU processes with the same coefficient $\alpha$. Because of the linearity of the stochastic differential equation satisfied 
by the $X_{i}$, the process $M_{n}$ is again an OU process starting at $x_{0}$, but with parameters $\alpha$ and $\sigma / \sqrt{n}$. Consequently, as $t$ tends to infinity it converges to its stationary distribution $v_{n}$, which is Gaussian with mean 0 and variance $\sigma^{2} / n$. Moreover, this convergence exhibits a cut-off phenomenon at the same instant as the sample. Let us denote by $\bar{d}_{n}(t)$ the total variation distance between the distribution of $M_{n}$ at time $t$ and its stationary distribution $v_{n}$ :

$$
\bar{d}_{n}(t)=\left\|\mathcal{L}\left(M_{n}(t)\right)-v_{n}\right\|_{\mathrm{TV}} .
$$

Proposition 2. The sequence $\left(t_{n}\right)_{n \in \mathbb{N}^{*}}$ is a sequence of cut-off instants for the convergence of the process $M_{n}$ to its stationary distribution, and, for all sufficiently large integers $n$ and for all real $u$ such that $t_{n}+u>0$, the following inequalities hold:

$$
\begin{aligned}
& 1-\exp \left(-\frac{x_{0}^{2}}{8 \sigma^{2}} \mathrm{e}^{-2 \alpha u}\right)+\bar{\varepsilon}_{n}(u) \leq \bar{d}_{n}\left(t_{n}+u\right), \\
& \bar{d}_{n}\left(t_{n}+u\right) \leq \sqrt{1-\exp \left(-\frac{x_{0}^{2}}{4 \sigma^{2}} \mathrm{e}^{-2 \alpha u}\right)}+\bar{\varepsilon}_{n}^{\prime}(u) .
\end{aligned}
$$

Here $\bar{\varepsilon}_{n}(u)$ and $\bar{\varepsilon}_{n}^{\prime}(u)$ converge to 0 as $n$ tends to infinity.

Notice that the sequence of cut-off instants is the same for the average of $n$ OU processes as it is for the sample. We might expect the convergence of the average to be faster than that of the sample, since the latter comprises a larger number of processes.

Proof of Proposition 2. As in the proof of Proposition 1, to ensure that $t_{n}$ is a cut-off instant in the sense of Definition 2 it suffices (in this case) to prove (7) and (8).

At time $t$, the average process $M_{n}$ has density $\mathcal{N}\left(x_{0} \mathrm{e}^{-\alpha t}, \sigma^{2}\left(1-\mathrm{e}^{-2 \alpha t}\right) / \sqrt{n}\right)$ and its stationary distribution, as $t$ tends to infinity, is $\mathcal{N}\left(0, \sigma^{2} / \sqrt{n}\right)$. Thus, the Hellinger distance between the distribution of $M_{n}$ at time $t$ and its stationary distribution can be computed using (4). As in the previous proof, inequality (3) then bounds the total variation distance by the Hellinger distance and the study of the behavior of the upper and lower bounds, as $n$ tends to infinity, gives the expected results.

Now we can see that the cut-offs for both the sample of OU processes and its average are very similar. The cut-off instants are the same and the tail behaviors have similar bounds. Let us now consider the total variation distance as a function of time: it decreases from 1 to 0 and, thus, $d_{n}$ (or $\bar{d}_{n}$ ) can be viewed as the survival function of a certain positive random variable concentrated around the cut-off instant $\log (n) /(2 \alpha)$. This (virtual) random variable could be viewed as 'the instant at which the sample reaches equilibrium'. As we shall see in the next section, the hitting time of 0 by the average process can be viewed as an approximation of this virtual instant.

\section{Hitting times}

Here, the link between the cut-off phenomenon and special hitting times will be explained. This idea has already appeared in [17], in the case of a sample $\left(X_{1}, \ldots, X_{n}\right)$ of finite-valued continuous-time Markov chains. Suppose that the sample converges to its stationary distribution. In [17], a cut-off was proved to occur at time

$$
\tau_{n}=\frac{\log (n)}{2 \beta},
$$


where $\beta$ is the spectral gap of the infinitesimal generator of the sampled process. The gap $\beta$ is unknown in general, and an estimator was proposed for it. For a certain functional $f$, the average process of the image, under $f$, of the sample is defined by

$$
M_{n}^{f}(t)=\frac{1}{n} \sum_{i=1}^{n} f\left(X_{i}(t)\right)
$$

The hitting time of the expected value of the stationary distribution of the sampled process by the average process $M_{n}^{f}$ is denoted by $T_{n}$. Ycart [17] proved that

$$
\lim _{n \rightarrow \infty} \frac{\mathrm{E}\left(T_{n}\right)}{\tau_{n}}=1
$$

and

$$
\lim _{n \rightarrow \infty} \frac{\operatorname{var}\left(T_{n}\right)}{\mathrm{E}\left(T_{n}^{2}\right)}=0,
$$

which shows that the random variable $\log (n) /\left(2 T_{n}\right)$ is a consistent estimator of $\beta$.

Let us apply the same idea to OU processes. Again consider a sample of $n$ OU processes starting at $x_{0}$ with parameters $\alpha$ and $\sigma$. We denote by $v$ their common stationary distribution, by $M_{n}$ the average process of the sample, defined as above, and take $f$ to be the identity function.

The distribution $v$ is centered (see Section 2.1); thus, the hitting time that we are interested in is the first instant at which the average process hits the level 0 , i.e.

$$
T_{0}^{x_{0}, n}=\inf \left\{t \geq 0: M_{n}(t)=0\right\} .
$$

As already seen, $M_{n}$ is an OU process starting at $x_{0}$ with parameters $\alpha$ and $\sigma / \sqrt{n}$, and the density $g_{n}$ of the hitting time $T_{0}^{x_{0}, n}$ can be deduced from (2) to be

$$
g_{n}(t)=\frac{\left|x_{0}\right|}{2 \sigma} \sqrt{\frac{n}{\pi \alpha}}\left(\frac{\alpha}{\sinh (\alpha t)}\right)^{3 / 2} \exp \left(-\frac{n x_{0}^{2} \mathrm{e}^{-\alpha t}}{4 \sigma^{2} \sinh (\alpha t)}+\frac{\alpha t}{2}\right) .
$$

From this explicit expression, the asymptotic distribution of $T_{0}^{x_{0}, n}$ can be computed.

Proposition 3. For a positive integer $n$, let $U_{n}$ be the following scaled version of $T_{0}^{x_{0}, n}$ :

$$
U_{n}=\alpha\left(T_{0}^{x_{0}, n}-\frac{\log (n)}{2 \alpha}+\frac{1}{2 \alpha} \log \left(\frac{2 \sigma^{2}}{x_{0}^{2}}\right)\right) .
$$

As $n$ tends to infinity, the distribution of $U_{n}$ converges weakly to the distribution on $\mathbb{R}$ with density $g$, where

$$
g(t)=\frac{2}{\sqrt{\pi}} \exp \left(-t-\mathrm{e}^{-2 t}\right)
$$

Proof. At time $t$, the density of the random variable $U_{n}$ is given by

$$
\frac{1}{\alpha} g_{n}\left(\frac{t}{\alpha}+\frac{\log (n)}{2 \alpha}-\frac{1}{2 \alpha} \log \left(\frac{2 \sigma^{2}}{x_{0}^{2}}\right)\right) .
$$

An elementary computation shows that, for all $t$,

$$
\lim _{n \rightarrow \infty} \frac{1}{\alpha} g_{n}\left(\frac{t}{\alpha}+\frac{\log (n)}{2 \alpha}-\frac{1}{2 \alpha} \log \left(\frac{2 \sigma^{2}}{x_{0}^{2}}\right)\right)=g(t) .
$$

Then Scheffé's lemma (see, e.g. [4, p. 223]) implies that the random variable $U_{n}$ converges in distribution to a random variable with density $g$. 
Proposition 3 implies that the random variable $T_{0}^{x_{0}, n}$ is concentrated around the cut-off instant $t_{n}$, in terms of convergence in probability:

$$
\lim _{n \rightarrow \infty} \frac{T_{0}^{x_{0}, n}}{\log (n) /(2 \alpha)}=1 \quad \text { in probability. }
$$

Thus, $\log (n) /\left(2 T_{0}^{x_{0}, n}\right)$ could be viewed as a consistent estimator of $\alpha$ if $\alpha$ were unknown.

Note also that the limit distribution $g$ can be viewed as the distribution of a random variable $Y$ defined by

$$
Y=-\log \left(\frac{|N|}{\sqrt{2}}\right),
$$

where $N$ is a standard Gaussian random variable. The distribution of $Y$ is asymmetrical and behaves in very different ways close to $-\infty$ and close to $\infty$. More precisely, if we denote by $\Phi$ the distribution function of the standard Gaussian distribution, we can see that the distribution function $G$ of the random variable $Y$ is given, for all real $y$, by

$$
G(y)=2\left(1-\Phi\left(\sqrt{2} \mathrm{e}^{-y}\right)\right) .
$$

The following approximations to the behaviors of $1-\Phi$ close to 0 and close to $\infty$ are well known (see [7, p. 175]):

$$
\begin{array}{ll}
1-\Phi(x) \sim \frac{1}{2}-\frac{x}{\sqrt{2 \pi}}, & x \rightarrow 0, \\
1-\Phi(x) \sim \frac{1}{x \sqrt{2 \pi}} \exp \left(-\frac{x^{2}}{2}\right), & x \rightarrow \infty .
\end{array}
$$

We therefore deduce the following approximations for the tail behaviors of $G$ at $\infty$ and $-\infty$ :

$$
\begin{aligned}
& G(y) \sim 1-\frac{2}{\sqrt{\pi}} \mathrm{e}^{-y}, \quad y \rightarrow \infty, \\
& G(y) \sim \frac{1}{\sqrt{\pi}} \exp \left(y-\mathrm{e}^{-2 y}\right), \quad y \rightarrow-\infty .
\end{aligned}
$$

For the convergences of both the sample and the average process, let us now compare the asymptotic tail behaviors of the hitting time $T_{0}^{x_{0}, n}$ to what happens before and after the cut-off instant.

After the cut-off instant $t_{n}$, the total variation distance decreases to 0 . The upper bounds of the total variation distances are (see Propositions 1 and 2)

$$
d_{n}\left(t_{n}+u\right) \leq \sqrt{1-\exp \left(-\frac{x_{0}^{2}}{4 \sigma^{2}} \mathrm{e}^{-2 \alpha u}\right)}+\varepsilon_{n}^{\prime}(u)
$$

and

$$
\bar{d}_{n}\left(t_{n}+u\right) \leq \sqrt{1-\exp \left(-\frac{x_{0}^{2}}{4 \sigma^{2}} \mathrm{e}^{-2 \alpha u}\right)}+\bar{\varepsilon}_{n}^{\prime}(u),
$$

where $\varepsilon_{n}^{\prime}(u)$ and $\bar{\varepsilon}_{n}^{\prime}(u)$ tend to 0 as $n$ tends to $\infty$. As $u$ tends to $\infty$, the upper bound in both cases is equivalent to

$$
\frac{\left|x_{0}\right|}{2 \sigma} \mathrm{e}^{-\alpha u}
$$


By Proposition 3, the right tail of $T_{0}^{x_{0}, n}$ can be evaluated as follows:

$$
\lim _{n \rightarrow \infty} \mathrm{P}\left(T_{0}^{x_{0}, n}>t_{n}+u\right)=1-G\left(\alpha u+\log \left(\frac{\sigma \sqrt{2}}{\left|x_{0}\right|}\right)\right) .
$$

Using the approximation of $G$ near $\infty$ (9), we deduce that

$$
1-G\left(\alpha u+\log \left(\frac{\sigma \sqrt{2}}{\left|x_{0}\right|}\right)\right) \sim \frac{\left|x_{0}\right|}{\sigma} \sqrt{\frac{2}{\pi}} \mathrm{e}^{-\alpha u}, \quad u \rightarrow \infty .
$$

The behavior of the right tail of $T_{0}^{x_{0}, n}$ thus matches that of the total variation distance following the cut-off instant, up to a constant factor $2 \sqrt{2 / \pi}$.

Before the cut-off instant $t_{n}$, the total variation distance increases (into the past) to 1 . By considering the lower bounds of the total variation distances evaluated at time $t_{n}-u$, where $u$ is a positive real number, we find that

$$
1-\exp \left(-\frac{x_{0}^{2}}{8 \sigma^{2}} \mathrm{e}^{2 \alpha u}\right)+\varepsilon_{n}(-u) \leq d_{n}\left(t_{n}-u\right)
$$

and

$$
1-\exp \left(-\frac{x_{0}^{2}}{8 \sigma^{2}} \mathrm{e}^{2 \alpha u}\right)+\bar{\varepsilon}_{n}(-u) \leq \bar{d}_{n}\left(t_{n}-u\right),
$$

where $\varepsilon_{n}(-u)$ and $\bar{\varepsilon}_{n}(-u)$ tend to 0 as $n$ tends to $\infty$. These lower bounds can be compared with the approximation of $\mathrm{P}\left(T_{0}^{x_{0}, n}<t_{n}-u\right)$. By Proposition 3 , the following convergence holds:

$$
\lim _{n \rightarrow \infty} \mathrm{P}\left(T_{0}^{x_{0}, n}<t_{n}-u\right)=G\left(-\alpha u+\log \left(\frac{\sigma \sqrt{2}}{\left|x_{0}\right|}\right)\right) .
$$

Using the approximation of $G$ near $-\infty(10)$, we deduce that

$$
G\left(-\alpha u+\log \left(\frac{\sigma \sqrt{2}}{\left|x_{0}\right|}\right)\right) \sim \frac{\sigma}{\left|x_{0}\right|} \sqrt{\frac{2}{\pi}} \exp \left(-\alpha u-\frac{x_{0}^{2}}{2 \sigma^{2}} \mathrm{e}^{2 \alpha u}\right), \quad u \rightarrow \infty .
$$

The agreement between the left tail of $T_{0}^{x_{0}, n}$ and the behavior of the total variation distance prior to the cut-off instant is not as good as that between the right tail and the behavior after the cut-off instant. However, the same type of doubly exponential tail is obtained in both cases.

Let us finish with a numerical illustration of the link between the cut-off phenomenon and the hitting time. We choose the parameters of the sampled OU process to be

$$
\alpha=1, \quad \sigma=1, \quad \text { and } \quad x_{0}=10 .
$$

We also fix the size $n$ of the sample, and we choose a sequence of regularly spaced instants $t_{1}, \ldots, t_{k}$. Our experiment involves simulating the sample of $n$ OU processes $\left(X_{1}, \ldots, X_{n}\right)$ with these parameters. At the instants $t_{1}, \ldots, t_{k}$, we compute the $p$-value of the Student $t$-test, which determines whether the sample $\left(X_{1}(t), \ldots, X_{n}(t)\right)$ has a centered Gaussian distribution with variance $\sigma^{2}$. We thus obtain a $k$-tuple of $p$-values. We also compute the values of the average process $M_{n}$ at each instant, and find the first time $T_{0}^{x_{0}, n}$ at which this process crosses the level 0 . We thus obtain one observation of this random variable.

We then repeat this experiment 1000 times, obtaining $1000 k$-tuples of $p$-values and 1000 observations of the random variable $T_{0}^{x_{0}, n}$. 
TABLE 1: Average of the $1000 p$-values of the Student $t$-test between the distribution of the sample and the stationary Gaussian distribution.

\begin{tabular}{cll}
\hline$t_{i}$ & $n=100$ & $n=1000$ \\
\hline 2.5 & 0 & 0 \\
3.0 & 0.00025 & 0 \\
3.5 & 0.017 & 0 \\
4.0 & 0.085 & 0.000016 \\
4.5 & 0.16 & 0.0076 \\
5.0 & 0.21 & 0.068 \\
5.5 & 0.23 & 0.15 \\
6.0 & 0.24 & 0.21 \\
\hline
\end{tabular}

TABLE 2: The $p$-values of the Kolmogorov-Smirnov test between the sample of the random variable $U_{n}$ and the limit distribution.

\begin{tabular}{rl}
\hline$n$ & $p$-value \\
\hline 100 & 0.0037 \\
1000 & 0.024 \\
\hline
\end{tabular}

We first compute the average of the $1000 p$-values for each instant $t_{1}, \ldots, t_{k}$. Table 1 shows the results obtained for $n=100$ and for $n=1000$, with $k=8$ in both cases.

For $n=100$, we can see that the Student $t$-test accepts the null hypothesis for instants between 3.5 and 4.5 , which means that the sample $\left(X_{1}(t), \ldots, X_{n}(t)\right)$ has a centered Gaussian distribution with variance $\sigma^{2}$, and, consequently, that the sample has converged. The theoretical value of the cut-off instant is $\log (100) /(2 \alpha)$, which is equal to 2.30 . For $n=1000$, the Student $t$ test accepts the null hypothesis for instants between 4.5 and 5 , which means that the convergence of the sample occurs between $t=4.5$ and $t=5$. Here, the theoretical value of the cut-off instant is $\log (1000) /(2 \alpha)$, which is equal to 3.45 .

Finally we consider the sample of 1000 observations of the random variable $T_{0}^{x_{0}, n}$. We want to illustrate the convergence in distribution proved in Proposition 3. We compute the scaled version of the sample,

$$
U_{n}=\alpha\left(T_{0}^{x_{0}, n}-\frac{\log (n)}{2 \alpha}+\frac{1}{2 \alpha} \log \left(\frac{2 \sigma^{2}}{x_{0}^{2}}\right)\right) .
$$

Then, a Kolmogorov-Smirnov test allows us to decide if the sample is distributed with the limit distribution. Table 2 shows the $p$-values that we obtain for this test.

We conclude that, at the $2 \%$ probability level, the Kolmogorov-Smirnov test accepts the null hypothesis for $n=1000$ but not for $n=100$.

\section{Concluding remarks}

We have proved that a cut-off phenomenon occurs during the convergence both of a sample of OU processes and its average, and at the same instant $t_{n}$ in each case. Moreover, we have found a sequence of hitting times $T_{0}^{x_{0}, n}$ that stay close to the cut-off instants as $n$ tends to infinity. Here the parameter $\alpha$ is known, but the random variable $\log (n) /\left(2 T_{0}^{x_{0}, n}\right)$ can be viewed as a 
consistent estimator of $\alpha$. It could be very useful in other models where a parameter is supposed to be unknown.

Finally, in future we would like to generalize this study to more general processes than the OU processes, and also to generalized average processes.

\section{Appendix A.}

Let us prove that the approximate total variation distance at time $t$ between the distribution of the one-dimensional OU process and its stationary distribution $v$ is given, as $t$ tends to $\infty$, by (1):

$$
\|\mathcal{L} X(t)-v\|_{\mathrm{TV}}=\frac{\left|x_{0}\right|}{\sigma \sqrt{2 \pi}} \mathrm{e}^{-\alpha t}+o\left(\mathrm{e}^{-2 \alpha t}\right)
$$

First, recall that both distributions, $\mathcal{L} X(t)$ and $v$, are Gaussian, i.e.

$$
\mathcal{L} X(t)=\mathcal{N}\left(x_{0} \mathrm{e}^{-\alpha t}, \sigma^{2}\left(1-\mathrm{e}^{-2 \alpha t}\right)\right), \quad v=\mathcal{N}\left(0, \sigma^{2}\right)
$$

If we denote by $u$ the quantity $\mathrm{e}^{-\alpha t}$, we have to estimate the total variation distance between $\mathcal{N}\left(x_{0} u, \sigma^{2}\left(1-u^{2}\right)\right)$ and $\mathcal{N}\left(0, \sigma^{2}\right)$ as $u$ tends to 0 . The total variation distance between two probability measures $\mathrm{P}$ and $\mathrm{Q}$ having respective densities $p$ and $q$ with respect to the same dominating measure $\lambda$ is given by (see, for instance, $[13$, p. 59])

$$
\|\mathrm{P}-\mathrm{Q}\|_{\mathrm{TV}}=\frac{1}{2} \int|p-q| \mathrm{d} \lambda
$$

In our case, we obtain

$$
\begin{aligned}
& \left\|\mathcal{L} X\left(\frac{-\log (u)}{\alpha}\right)-v\right\|_{\mathrm{TV}} \\
& \quad=\frac{1}{2} \int_{\mathbb{R}}\left|\frac{1}{\sigma \sqrt{2 \pi}} \mathrm{e}^{-x^{2} /\left(2 \sigma^{2}\right)}-\frac{1}{\sigma \sqrt{2 \pi} \sqrt{1-u^{2}}} \exp \left(-\frac{\left(x-x_{0} u\right)^{2}}{2 \sigma^{2}\left(1-u^{2}\right)}\right)\right| \mathrm{d} x,
\end{aligned}
$$

which can be rewritten as

$$
\left\|\mathcal{L} X\left(\frac{-\log (u)}{\alpha}\right)-v\right\|_{\mathrm{TV}}=\frac{1}{2} \mathrm{E}\left|1-\frac{1}{\sqrt{1-u^{2}}} \exp \left(\frac{x_{0}^{2}}{2 \sigma^{2}}\right) \exp \left(-\frac{\left(N-x_{0} /(\sigma u)\right)^{2}}{2\left(1 / u^{2}-1\right)}\right)\right|
$$

where $N$ is a standard Gaussian random variable.

Considering the function inside the expectation as a function of $u$, we compute its polynomial approximation as $u$ tends to 0 using the Taylor-Lagrange formula, to obtain

$$
\left|1-\frac{1}{\sqrt{1-u^{2}}} \exp \left(\frac{x_{0}^{2}}{2 \sigma^{2}}\right) \exp \left(-\frac{\left(N-x_{0} /(\sigma u)\right)^{2}}{2\left(1 / u^{2}-1\right)}\right)\right|=\frac{\left|x_{0} u N\right|}{\sigma}+u^{2} \epsilon(u, N),
$$

where $\epsilon$ is a function polynomial in $N$ that tends to 0 as $u$ tends to 0 .

Taking the expectation, and knowing that all the moments of the standard Gaussian random variable are finite, we obtain

$$
\|\mathcal{L} X(t)-v\|_{\mathrm{TV}}=\frac{\left|x_{0}\right| \mathrm{e}^{-\alpha t}}{\sigma \sqrt{2 \pi}}+o\left(\mathrm{e}^{-2 \alpha t}\right)
$$

which is the expected result. 


\section{References}

[1] Alili, L., Patie, P. and Pedersen, J. (2005). Representations of the first hitting time of an Ornstein-Uhlenbeck process. To appear in Stoch. Models.

[2] Barrera, J., LACHAUd, B. AND YCART, B. (2005). Cutoff for $n$-tuples of exponentially converging processes. Submitted.

[3] Bharucha-ReID, A. (1960). Elements of The Theory of Stochastic Processes and Their Applications. McGrawHill, New York.

[4] Billingsley, P. (1968). Convergence of Probability Measures. John Wiley, New York.

[5] Diaconis, P. (1996). The cut-off phenomenon in finite Markov chains. Proc. Nat. Acad. Sci. USA 93, $1659-1664$.

[6] Diaconis, P., Graham, R. and Morrison, J. (1990). Asymptotic analysis of a random walk on a hypercube with many dimensions. Random Structures Algorithms 1, 51-72.

[7] Feller, W. (1968). An Introduction to Probability Theory and its Applications, Vol. 1, 3rd edn. John Wiley, New York.

[8] Feller, W. (1971). An Introduction to Probability Theory and Its Applications, Vol. 2, 2nd edn. John Wiley, New York.

[9] Jeanblanc, M. And RutKowski, M. (2000). Modelling of default risk: an overview. In Modern Mathematical Finance: Theory and Practice, Higher Education Press, Beijing, pp. 171-269.

[10] Lansky, P., Sacerdote, L. and Tomasetti, F. (1995). On the comparison of Feller and Ornstein-Uhlenbeck models for neural activity. Biol. Cybernetics 73, 457-465.

[11] Leblanc, B. And Scaillet, O. (1998). Path dependent options on yields in the affine term structure. Finance Stoch. 2, 349-367.

[12] Pitman, J. and Yor, M. (1981). Bessel processes and infinitely divisible laws. In Stochastic Integrals (Proc. Symp. Univ. Durham, 1980; Lecture Notes Math. 851), Springer, Berlin, pp. 285-370.

[13] Pollard, D. (2001). A User's Guide to Measure Theoretic Probability. Cambridge University Press.

[14] Saloff-Coste, L. (1997). Lectures on finite Markov chains. In Lectures on Probability Theory and Statistics (Saint-Flour, 1996; Lecture Notes Math. 1665), Springer, Berlin, pp. 301-413.

[15] Saloff-Coste, L. (2004). Random walks on finite groups. In Probability on Discrete Structures (Encyclopaedia Math. Sci. 110), ed. H. Kesten, Springer, Berlin, pp. 263-346.

[16] Uhlenbeck, G. And Ornstein, L. (1930). On the theory of Brownian motion. Phys. Rev. 36, 823-841.

[17] YCART, B. (1999). Cutoff for samples of Markov chains. ESAIM Prob. Statist. 3, 89-107.

[18] YCART, B. (2001). Cutoff for Markov chains: some examples and applications. In Complex Systems, eds E. Goles and S. Martínez, Kluwer, Dordrecht, pp. 261-300. 